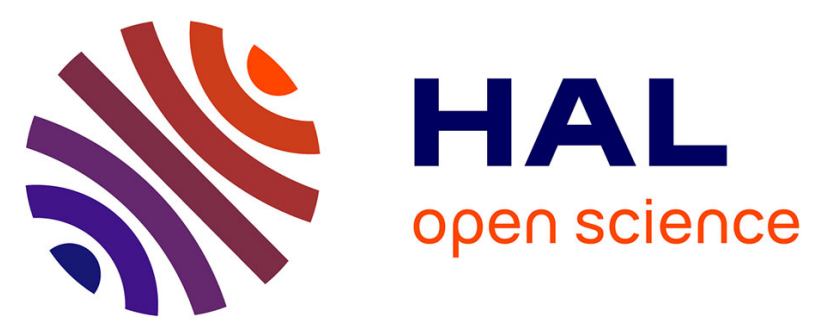

\title{
Fusion of 3D real-time echocardiography and cine MRI using a saliency analysis
}

Angélica Atehortúa, Mireille Garreau, Antoine Simon, Erwan Donal, Mathieu

Lederlin, Eduardo Romero

\section{- To cite this version:}

Angélica Atehortúa, Mireille Garreau, Antoine Simon, Erwan Donal, Mathieu Lederlin, et al.. Fusion of 3D real-time echocardiography and cine MRI using a saliency analysis. International Journal of Computer Assisted Radiology and Surgery, 2020, 15 (2), pp.277-285. 10.1007/s11548-019-02087-w . hal-02364096

\section{HAL Id: hal-02364096 \\ https://hal-univ-rennes1.archives-ouvertes.fr/hal-02364096}

Submitted on 11 Dec 2019

HAL is a multi-disciplinary open access archive for the deposit and dissemination of scientific research documents, whether they are published or not. The documents may come from teaching and research institutions in France or abroad, or from public or private research centers.
L'archive ouverte pluridisciplinaire HAL, est destinée au dépôt et à la diffusion de documents scientifiques de niveau recherche, publiés ou non, émanant des établissements d'enseignement et de recherche français ou étrangers, des laboratoires publics ou privés. 


\title{
Fusion of 3D real-time echocardiography and cine MRI using a saliency analysis
}

\author{
Angélica Atehortúa - Mireille Garreau • \\ Antoine Simon · Erwan Donal · Mathieu \\ 5 Lederlin - Eduardo Romero
}

7 Received: date / Accepted: date

\begin{abstract}
8 Abstract

\section{Purpose:}

- This paper presents a novel 3D multimodal registration strategy to fuse 3D 1 real-time echocardiography images with cardiac cine MRI images. This align12 ment is performed in a saliency space, designed to maximize similarity between 13 the two imaging modalities. This fusion improves the quality of the available 4 information.

15 Methods:

${ }_{16}$ The method performs in two steps: temporal and spatial registrations. A tem17 poral alignment is firstly achieved by non-linearly matching pairs of correspon18 dences between the two modalities using a dynamic time warping. A temporal 19 registration is then carried out by applying non-rigid transformations in a 20 common saliency space where normalized cross correlation between temporal 21 pairs of salient volumes is maximized.

22 Results:

23 The alignment performance was evaluated with a set of 18 subjects, 3 with 24 cardiomyopathies and 15 healthy, by computing the Dice score and Haus25 dorff distance with respect to manual delineations of the left ventricle cavity 26 in both modalities. A Dice score and Hausdorff distance of $0.86 \pm 0.04$ and ${ }_{27} \quad 13.61 \pm 3.86 \mathrm{~mm}$ respectively were obtained. In addition, the deformation field
\end{abstract}

A. Atehortúa (orcidID 0000-0002-6192-1757)

Univ Rennes, CHU Rennes, Inserm, LTSI - UMR 1099, F-35000 Rennes, France

Tel.: + 330223236220

E-mail: amatehortual@unal.edu.co

M. Garreau · A. Simon (orcidID 0000-0001-6023-6427) · E. Donal (orcidID 0000-0002-90831582) · M. Lederlin (orcidID 0000-0003-1388-5277)

Univ Rennes, CHU Rennes, Inserm, LTSI - UMR 1099, F-35000 Rennes, France

E-mail: \{mireille.garreau,antoine.simon,erwan.donal,mathieu.lederlin\}@univ-rennes1.fr

A. Atehortúa · E. Romero (orcidID 0000-0003-2088-2509)

Universidad Nacional de Colombia, Bogotá, Colombia

E-mail: edromero@unal.edu.co 
was estimated by quantifying its foldings, obtaining a $98 \%$ of regularity in the deformation field.

\section{Conclusions:}

The 3D multimodal registration strategy presented is performed in a saliency space. Unlike state of the art methods, the presented one takes advantage of the temporal information of the heart to construct this common space, ending up with two well aligned modalities and regular deformation fields. This preliminary study was evaluated on heterogeneous data composed by two different datasets, healthy and pathological cases, showing similar performances in both cases. Future work will focus on testing the presented strategy in a larger dataset with a balanced number of classes.

Keywords Image registration · spatio-temporal saliency · cardiac cine MRI images · echocardiography

\section{Introduction}

Cardiac imaging plays an important role in diagnosis and treatment planning of prevalent heart diseases. Non-invasive procedures, specifically $3 \mathrm{D}$ real-time echocardiography (3DUS $+\mathrm{t}$ ) and cine cardiac MRI (cMRI), provide structural and functional information of the heart. While $3 \mathrm{DUS}+\mathrm{t}$ is a first option for cardiac evaluation and follow-up, cMRI is used when a diagnosis doubt persists after the ultrasound examination [9]. In pathologies such as pulmonary arterial hypertension, ischaemic heart disease, arrhythmogenic ventricular cardiomyopathy and atrial arrhythmias [24,18,2], the information obtained with these two modalities results to be complementary. Under these considerations, multimodal image fusion should provide a more accurate and reliable examination by combining morphological (cMRI) and functional (3DUS+t) assessments $[18,24]$. This fusion could facilitate anatomo-functional correlations of cardiac abnormalities that lead to design new diagnostic and follow-up markers $[6,17,2]$ to detect early impairments and subtle changes in the cardiac function. Consequently, a better therapy follow-up could be performed to improve patient categorization, personalized treatment and survival.

Image fusion is generally carried out by a registration process [12]. However, a useful registration between 3DUS+t and MRI images is a complex and difficult process, basically because the represented information is captured using very different physical sensors. Furthermore, 3DUS+t images have a lower signal-to-noise ratio (SNR) than $2 \mathrm{DUS}+\mathrm{t}$ images. There are also several factors that increase a poor definition of the left ventricular chamber and walls during the acquisition in echocardiography, such as anatomic particularities (obesity, asthenic habitus) and other related information, such as acquired disease that affects the acoustic window.

Few works in the state of the art have addressed the challenge of registering 3D echocardiography and cine MRI images. These approaches can be divided in two groups: intensity-based registration $[10,28,13]$ and surfaced-based registration $[15,20]$. In the first group, works map MRI and 3DUS modalities to a 
common space using only spatial information. Some of them transformed both modalities into their corresponding phase maps using Fourier transform [28].

However, in some cases, this phase representation is very similar to the original image and may lead to erroneous registrations. Other works $[10,13,27]$ align the modalities in the intensity space, where the registration is prone to error due to the high data heterogeneity and the noisy US images. For the second group, surfaced-based registration, cardiac structures are segmented in both modalities and then, a surface extracted from one modality is non rigidly-registered to the other surface modality. In this group, strategies based on the registration of common landmarks in both modalities are also taken into account. Nevertheless, these approaches are highly sensitive to the segmentation and precise setting of landmarks, due to the inter- and intra-shape variability of the heart, its complex dynamic and the image quality.

Unlike these works, which are based only on spatial registration or surface deformations, this paper proposes a novel 3D multimodal registration framework by combining saliency and temporal information, which is applied to cMRI and 3DUS+t images. In this work, a non rigid deformation is performed to cope with local differences between images, since that heart is intrinsically deformable, even if intra-patient cases are considered. Although a preliminary work of this study has been previously published as a conference paper [30], this paper is substantially different from that work in that it incorporates an initial registration to perform a more robust alignment between both modalities and it includes more experimental validation results and the use of quantitative metrics for the evaluation.

The paper is organized as follows: In Section 2 the registration framework is described. Experiments and results are provided in Section 3. Finally, conclusions are presented in Section 4.

\section{Methodology}

Each image modality (cMRI noted as $M R$ and 3DUS+t noted as $U S$ ) is represented as a sequence $I(x, y, z, t)$ of images, where $(x, y, z) \in \Omega_{I}$ corresponds to the spatial coordinates in the domain of the image $\left(\Omega_{I}\right)$ and $t \in\{1, T\}$ represents a temporal position in the sequence, being $T$ the number of frames per cardiac cycle for each modality, MR and US, which can be different, i.e, $T^{m r}$ and $T^{u s}$. This work proposes a $3 \mathrm{D}$ registration strategy to map each voxel of the moving modality to its corresponding voxel in the reference modality. The registration process is decoupled into two temporal and spatial steps of alignment, that are hereafter described.

\subsection{Temporal alignment}

Firstly, a temporal alignment sets the frame correspondences between both image sequences. For doing so, two sub-steps are performed: i) an image- 
based cardiac dynamic descriptor and ii) a nonlinear mapping between both modalities, using the method proposed by Betancur et al [4].

\subsubsection{Image-based cardiac dynamic descriptor.}

A $\Phi(t ; I)$ curve that describes events of the cardiac cycle consistently $[19$, $27,4]$ is constructed using all volumes or image sequence $I_{t}$ of the cardiac cycle. This process is independently performed for each image sequence $I_{t}$, by computing the similarity between the volume at the end of the diastole (ED) with respect to each of the remaining volumes of the cycle, Eq. 1. The ED frame is arbitrarily set to the first frame of the sequence, $I_{t=1}$. The classical normalized cross correlation is used as similarity measure since this measure is less sensitive to different noisy conditions.

$$
\Phi\left(t ; I_{t}\right)=\left\langle\frac{I_{t=1}-\bar{I}_{t=1}}{\left\|I_{t=1}-\bar{I}_{t=1}\right\|}, \frac{I_{t}-\bar{I}_{t}}{\left\|I_{t}-\bar{I}_{t}\right\|}\right\rangle \quad \forall t \in\{1, T\}
$$

being $\overline{I_{t}}$ the average intensity value of the image $I_{t}$.

\subsubsection{Nonlinear mapping between the images in the reduced space.}

Due to the non-linear nature of the cardiac function, a dynamic time warping (DTW) [3] is applied to map each point of the curve $\Phi^{m r}(t)$ to its corresponding point of the curve $\Phi^{u s}(t)$, being $\Phi(t)$ the curve described in equation 1 . As a result of this mapping, a set of aligned temporal pairs $A=\left\{\left(t_{i}^{u s}, t_{j}^{m r}\right)\right\}$ is obtained for each subject. Despite that several US volumes could be matched with the same MR volume or the other way around, this does not pose any issue because this temporal alignment matches volumes that are close in time and from the same cardiac phase.

\subsection{Spatial alignment}

A main contribution of this work is to spatially register the image sequences by using spatio-temporal information of the cardiac function in the $3 \mathrm{D}$ domain. For doing such registration, two sub-steps are performed: i) mapping the image sequence to a common saliency space and ii) setting customized registration metric in the saliency space.

\subsubsection{Mapping the image sequence to a saliency space.}

Multimodal cardiac images are statistically dependent by the fact of being captured from the very same object, i.e., the heart, and such dependency could be used to fuse them. The human visual system (HVS) is able to extract relevant information by decomposing the scene into low level representations which are integrated and encoded as visual information [22]. Under such hypotheses, the 
salient volume is built upon a multi-scale region-based decomposition which preserves the most correlated information and is obtained by applying a classic Gaussian pyramidal filtering [5]. For every image sequence, five scales $s$ are taken, namely the original scale, a finer level and three incrementally coarser levels of the original. The finer level provides more details of the heart, while the coarser levels provide a global localization of the heart. For each scale $s$, regions $r$ are extracted by using the simple linear iterative clustering (SLIC) algorithm [1]. The Cartesian coordinates of these regions are stored as a grid $G$ since every processing is from here on performed exclusively within this grid. Afterwards, each image is filtered for each scale $s$ and for each instant of the time $t$ by applying steerable filters [7]. These filters correspond to an oriented first derivative of a 3D Gaussian with a standard deviation set to 3, due to the noise in cMRI and 3DUS images can be modeled by Gaussian or Laplacian distribution [29]. The result of this bank of filters is an image $S V_{t}$ for each scale $s$ where their structural features, such as intensity, orientation and edges, are enhanced, while it reduces noise.

Saliency is defined by a consistent difference between regional $r$ features and their surroundings during the time, Eq. 2. Independently of each scale $s$, the salient volume at time $t$ is estimated by comparing it with respect to the remaining frames. This ensures temporal coherence of the salient region, even in scenes with a higher level of complexity, such as low frame rate and noisier sequences $[11,26]$.

$$
\operatorname{saliency}(t, r, s)=\sum_{\tau \in T}\left|S V_{t}(r, s)-S V_{\tau}(r, s)\right|^{2} \quad \forall r \in G
$$

The final salient volume $S$ is obtained by averaging saliency for every scale, previously set to the original size either by interpolation or decimation. This set of salient volumes constitutes the saliency space. Figures 1 and 2 show examples of saliency measured in echocardiography and magnetic resonance imaging respectively. Observe how the myocardium is highlighted in both modalities. This representation provides a common space where both image modalities can be spatially aligned.
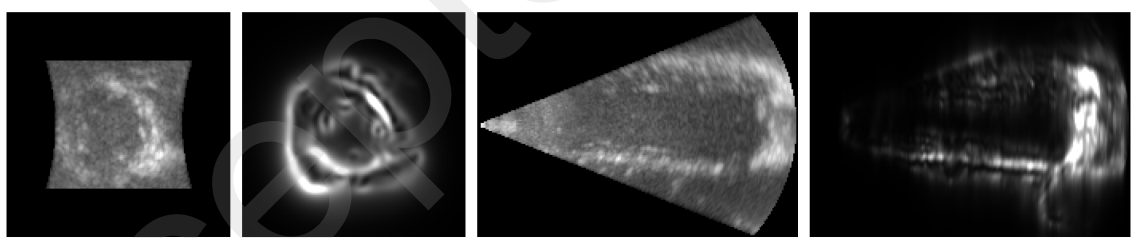

Fig. 1 Salient images in echocardiography. Panels from left to right: original image in axial view, salient image in axial view, original image in coronal view and salient image in coronal view. 


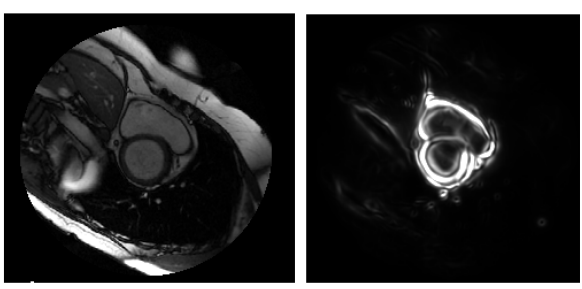

Fig. 2 Salient image in short axis cine MRI. Left panels shows the original image and the right panel illustrates its respective saliency.

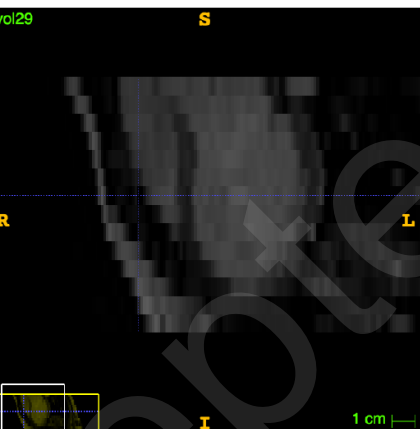

(a) Original image tructures of interest [16].

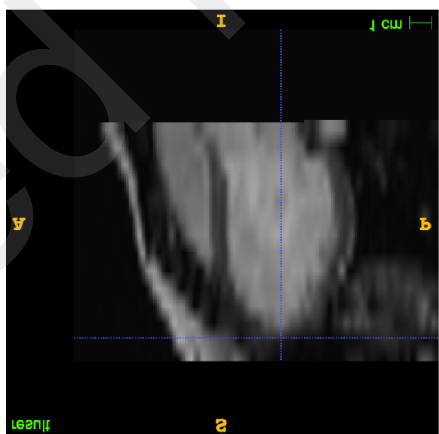

(b) Resampled MR volume

Fig. 3 Example of a resampled MRI volume in the longitudinal direction.

\subsubsection{Setting customized registration metric in the saliency space.}

Since the field of view is not the same in both modalities, a coarse manual delineation of the epicardium of the left ventricle (LV) at the end of the diastole (ED) is obtained to delimit a common region of interest (ROI). Herein, this LV ROI is used for the whole cardiac cycle, under the hypothesis that myocardium contracts towards the center of the LV and such ROI encloses all the cardiac

The intensity image sequence $I_{t}$, the LV ROI and the saliency image $S$ are oriented to the same Right-Anterior-Inferior radiological coordinates $R A I$, defining a common $x, y, z$ order, using the ITK-snap tool [25]. This orientation only describes the mapping from the voxel coordinate system to the physicoordinate system. As 3DUS+t modality has a higher spatial resolution n cMRI, the oriented LV ROI, the salient volume and the intensity imfrom cMRI modality are linearly resampled to obtain the sam dimensions and voxel size of the echocardiographic image. Figure 3 shows a resampled MRI image for a healthy subject in the longitudinal direction.

Fig. 3 Example of a resampled MRI volume in the longitudinal direction.

Afterwards, a spatial registration is performed in the saliency space delimited by the LV ROI. For each temporal pair $\left(t_{i}^{u s}, t_{j}^{m r}\right) \in A$ obtained as described in section 2.1, a mapping-salient function $\mathcal{F}_{\hat{t}}$ sets each voxel in the 
cMRI image to its corresponding voxel in the 3DUS domain at the instant of time $\hat{t}=\left(t_{i}^{u s}, t_{j}^{m r}\right)$. The mapping-salient function for each temporal pair is then defined as:

$$
\mathcal{F}_{\hat{t}}\left(S_{t_{i}}^{u s}, S_{t_{j}}^{m r}\right)=\underset{T_{x}}{\operatorname{argmax}} \operatorname{sim}\left(S_{t_{i}}^{u s}, S_{t_{j}}^{m r}\left(T_{x}\right) ; T_{x}\right)
$$

where $T_{x}$ is a transformation model that maximizes the similarity ( $\mathrm{sim}$ ) between both salient volumes, $S_{t_{i}}^{u s}$ and $S_{t_{j}}^{m r}$. In this work, $T_{x}$ is a non-rigid transformation applied to the salient volumes via interpolation with a B-spline of order 3 that maximizes the normalized cross correlation. This similarity measure is used since it is invariant to affine intensity distortions [8]. Herein, an adaptive stochastic gradient descent [14] was used as optimizer. In order to achieve an accurate alignment, this non-rigid registration was initialized by an Eulerian transformation [21] that rigidly aligns the LV ROI centroids of both modalities.

Once the mapping-salient function $\mathcal{F}_{\hat{t}}$ is obtained for each temporal pair $\hat{t}$, it is applied to the original image sequence cMRI, $I_{t_{j}}^{m r}\left(\mathcal{F}_{\hat{t}}\right)$, mapping it to the $3 \mathrm{D}$ echocardiography image domain $I_{t_{i}}^{u s}$. This process is applied for all the aligned temporal pairs and in consequence, the whole cMRI sequence is covered.

\section{Experiments and results}

\subsection{Image Data}

Two datasets of pairs of cMRI and 3DUS+t data were used: healthy subjects and pathological subjects.

\subsubsection{Healthy group.}

This group comprises 15 healthy subjects [23]. cMRI and 3DUS+t acquisitions were obtained within an interval of $3.5 \pm 3.3$ days. Acquisition features for cMRI and 3DUS+t are, respectively: temporal resolution of 30 and $14.93 \pm$ 3.03 frames per second; slice thickness of $8 \mathrm{~mm}$ and $0.71 \pm 0.09 \mathrm{~mm}$; and inplane resolution voxel size of $1.22 \pm 0.05 \mathrm{~mm}$ and $0.84 \pm 0.16 \mathrm{~mm}$. Delineated volumes of the left ventricle cavity are provided with each modality for all the cardiac cycle.

\subsubsection{Pathological group.}

It is composed of 3 subjects with dilated and hypertrophic cardiomyopathy, who followed an acquisition protocol comprising both cMRI and 3DUS+t. This dataset was acquired at CHU-Pontchaillou in Rennes, France. This study was part of a systematic database review conducted following the Declaration of Helsinki and approved under the CNIL (National Commission on Informatics 
and Liberty of France). Acquisition features for cine MRI and 3DUS+t were, respectively: temporal resolution of $28.33 \pm 5.77$ and $22.33 \pm 14.98$ frames per second; slice thickness of $8 \mathrm{~mm}$ and $0.74 \pm 0.11 \mathrm{~mm}$; and in-plane resolution voxel size of $1.62 \pm 0.1231 \mathrm{~mm}$ and $1.08 \pm 0.15 \mathrm{~mm}$. Delineated volumes of the left ventricle cavity are manually provided in each modality at the end of the diastole and end of the systole.

The method performance was evaluated for each step, temporal and spatial alignment, as follows.

\subsection{Evaluation of the temporal alignment}

The temporal alignment was validated for each image modality and dataset by computing the Mean Absolute Error (MAE) between the time point corresponding to the end of the systole (ES), which was manually set by an experienced observer, and the time point with the minimum value of the curve $\Phi$ obtained in Section 2.1.1. This MAE measures the distance in number of frames between the ES position estimated by the method and the position set by the expert. The MAE for the healthy group was $2.73 \pm 1.67$ frames and 0.93 \pm 0.70 frames for cMRI and 3DUS+t, respectively. While the MAE for the pathological group was 1.0 1.0 frames and $2.33 \pm 1.53$ frames for cMRI and 3DUS+t, respectively.

\subsection{Evaluation of the spatial alignment}

The results differ according to the temporal duration and the phases that were considered in the saliency estimation. Different tests have been conducted, along the heart cycle providing the results presented in the paper and along each cardiac period (diastole and systole) providing some differences. These second results showed a slight difference in accuracy about the $5 \%$. In this sense, the complete cardiac cycle is used to compute the saliency in each modality.

Qualitative results of the spatial alignment are illustrated for one pathological case in Fig. 4, from the base to the apex (in rows) at four instants of time presented in the columns (End of the diastole ED, end of the systole ES, intermediate time between the ED and ES, and intermediate time between the ES and the final frame). Observe the good spatial alignment between cMRI (green color) and 3DUS+t (magenta color) volumes using the saliency descriptor and the registration framework, in particular for the basal, basal-mid and mid views, i.e, the myocardium is good overlapped in both modalities.

To quantitatively evaluate the registration performance, aligned delineations of the LV cavity from both modalities were compared at the ED and ES by computing the Dice Score and Hausdorff distance.

Table 1 and Fig. 5 show the spatial registration performance of the presented strategy, when it is evaluated in terms of the Dice score and Hausdorff 

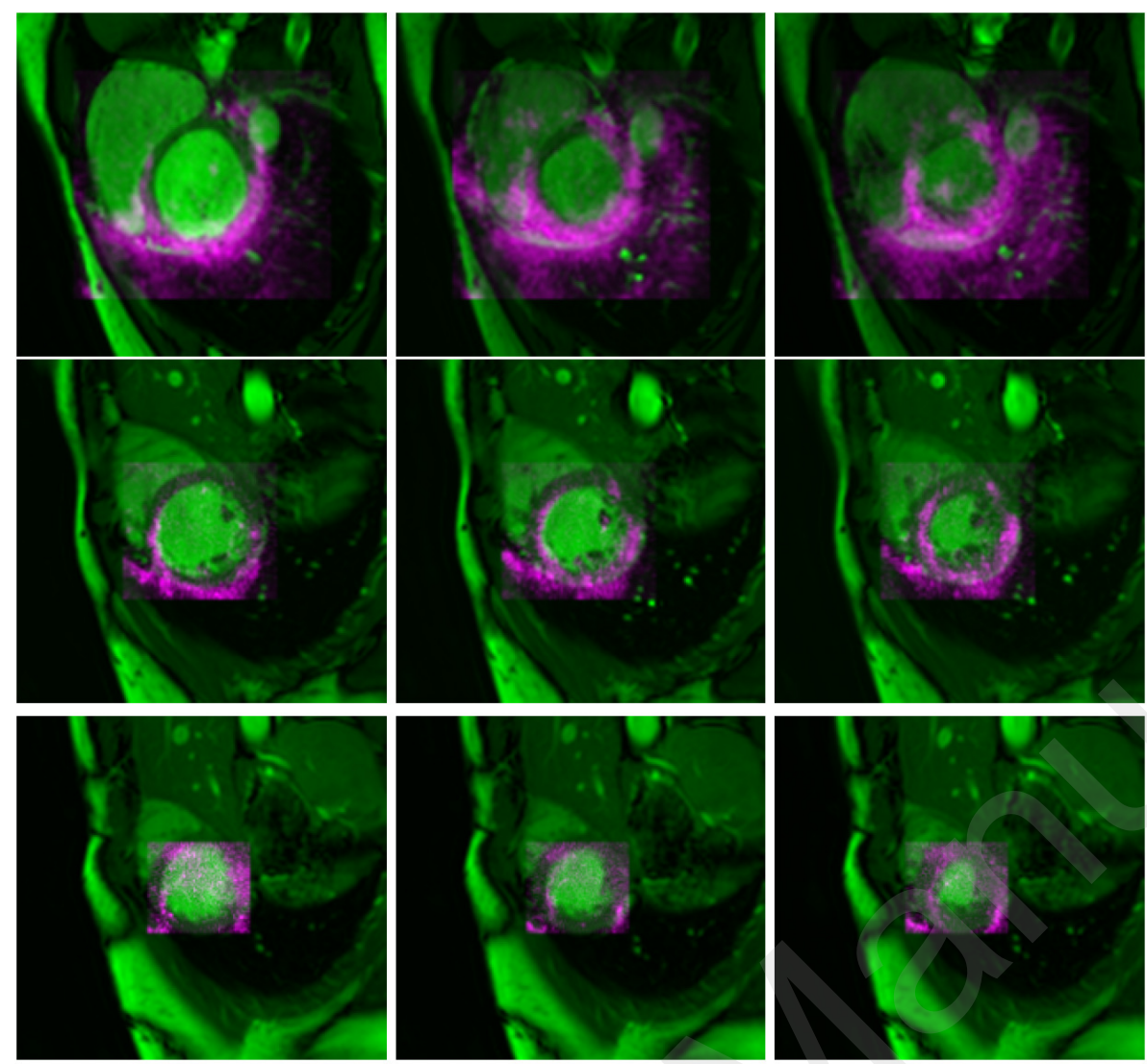

Fig. 4 Illustration of a spatial alignment for one pathological case. MRI (green color) superimposed to the 3DUS (magenta color). Rows correspond to the volume levels, from top to bottom: Basal, mid and apex-mid. Columns correspond to the instants of time, from left to right: end of the diastole ED, end of the systole ES and intermediate time between ED and ES.

distance. The method achieves accurate correspondences between both modalities with an average Dice score higher than 0.8 , a level that could be clinically acceptable. However, a registration method can be useless if there are too many foldings in the deformation field, even for cases of good match. Hence, regularity of the deformation field, for the whole dataset, was evaluated by computing the number of voxels for which the determinant of the Jacobian is non-positive. This analysis showed only $0.2 \pm 0.3 \%$ of the voxels exhibited such values. In addition, registration with the elastix [31] software corresponded to the nonrigid B-spline method [35,34], which has been described to robustly operate in similar problems $[32,33]$.

In addition, the benefit of this method has been evaluated by computing the Dice Score and the Hausdorff distance at each step of the method to see 
Table 1 Spatial registration performance, for healthy and pathological groups, by computing the Dice score and the Hausdorff distance

\begin{tabular}{lll}
\hline Measure & Healthy & Pathological \\
\hline Dice Score & $0.88 \pm 0.03$ & $0.83 \pm 0.08$ \\
Hausdorff distance $(\mathbf{m m})$ & $12.02 \pm 1.36$ & $15.20 \pm 2.93$ \\
\hline
\end{tabular}

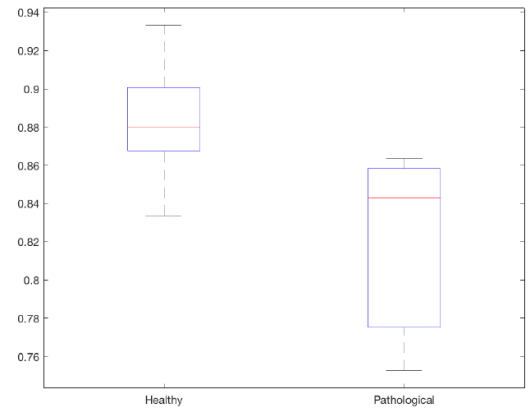

(a) Dice Score

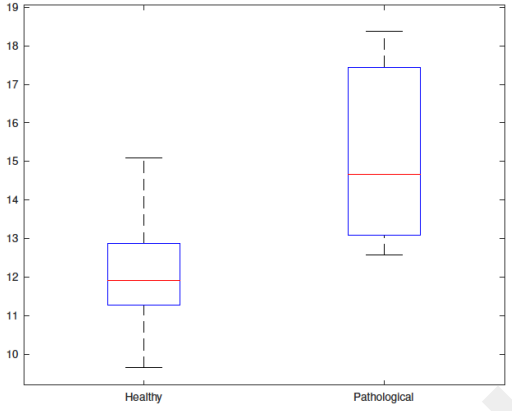

(b) Hausdorff distance

Fig. 5 Boxplot that shows the minimum and maximum values for a) Dice Score and b) Hausdorff distance computed in the healthy and pathological datasets

the contribution of the registration step in the saliency space. The results are presented in Table 2. Columns show the results about the type of transformations applied in the saliency space. The first column represents the comparison of the LV surfaces without performing any transformation, i.,e, the original LV surfaces compared between them. The remaining columns are then results of the initial, affine and non-rigid transformations applied to the salient volumes. Note how, the Dice Score value passed from 0.40 to 0.76 after applying the initial rigid transformation and then, the registration performance improved to 0.88 , when the final stage, non-rigid registration, was performed. The same observations can be made for the Hausdorff distance, in which a value of $13.01 \mathrm{~mm}$ was obtained after applying the initial registration, while a value of $12.02 \mathrm{~mm}$ was estimated at the final stage.

Table 2 Spatial registration performance in each transformation: without any transformation (without), initial registration and final registration (affine and non-rigid), using the healthy dataset

\begin{tabular}{ccccc}
\hline Measure & & \multicolumn{2}{c}{ Transformation } & \\
& Without & Initial & Affine & Nonrigid \\
\hline Dice Score & $0.40 \pm 0.13$ & $0.76 \pm 0.7$ & $0.80 \pm 0.05$ & $0.88 \pm 0.03$ \\
Hausdorff distance $(\mathbf{m m})$ & $15.30 \pm 13.40$ & $13.01 \pm 0.13$ & $12.89 \pm 0.13$ & $12.02 \pm 1.36$ \\
\hline
\end{tabular}


Table 3 Spatial registration performance in the intensity space, for healthy and pathological groups, by computing the Dice score and the Hausdorff distance

\begin{tabular}{lll}
\hline Measure & Healthy & Pathological \\
Dice Score & $0.77 \pm 0.05$ & $0.79 \pm 0.08$ \\
Hausdorff distance $(\mathbf{m m})$ & $19.34 \pm 3.67$ & $19.32 \pm 4.04$ \\
\hline
\end{tabular}

Moreover, two additional experiments to emphasize the advantage of the method were performed. The first experiment consisted of aligning the intensity images using the same process computed for the salient volumes. The results are presented in Table 3, showing that the Dice Score measure was of 0.77 for 15 healthy cases and 0.79 for pathological cases, in comparison with the presented method, 0.88 and 0.83 for healthy and pathological subjects, respectively. In a similar way, the Hausdorff distance results were of $19.34 \mathrm{~mm}$ and $19.32 \mathrm{~mm}$ for healthy and pathological subjects, while $12.02 \mathrm{~m}$ and $15.20 \mathrm{~m}$ for the presented strategy. These results highlight the advantage of using the saliency space to align the modalities. The second experiment consisted of adding Gaussian noise (with variance of 0.02) to the intensity images and after applying the registration in the saliency space. For this experiment, a subset composed by five randomly selected healthy subjects was tested, reporting a lower value of the Dice score and Hausdorff distance about $10 \%$ with respect to the results shown in Table 1 . These results suggest the saliency in echocardiography is affected by the noise, due to the fact that cardiac structures can not be well recognized by fuzzy edges. This could be mitigated by performing a robust 3D denoising algorithm that improves the image quality in echocardiography.

As mentioned in the introduction, this paper is substantially different to the work published in [30], since a more detailed evaluation was performed and a larger dataset was used. In addition, the Dice score was computed for the resulting aligned LV surfaces from pathological subjects using the registration strategy described in that published work. A Dice score of 0.78 was reported in that published work, while the presented method outperforms such work with a Dice score of 0.83 , about $7 \%$.. The advantages from this new work is the use of a 3D saliency, which is applied to each modality and the initial registration. Unlike the previous work, which was performed using a 2D approach and without any initial alignment.

\section{Conclusions}

A novel 3D multimodal registration method was proposed. This strategy includes the temporal information of the heart to obtain a common space of reference based on a saliency measure, where both modalities are aligned. The method has been tested on 15 healthy cases and 3 patients with cardiomyopathies. The evaluation has been realized by a visual and qualitative 
way and by a quantitative strategy using Dice Score and 3D Hausdorff measures. Results have shown an interest of this method based on registration in saliency space by comparison to a registration approach based only on intensity. In addition, the results show an improvement when the initial rigid registration step results are compared with the results obtained at the final stage by using the saliency. Results have shown a good alignment between $\mathrm{cMRI}$ and 3DUS+t, even if the method has to be evaluated in a quantitative way on a large database. Future research will focus on improving the 3DUS image quality to obtain more reliable region of interest of the left ventricle. In addition, a rigid transformation between the modalities in the saliency space will be explored to study the effects of the heart deformation by our algorithm. The presented strategy will be tested with a larger dataset of healthy and pathological subjects and will be compared with other methods of the literature.

\section{Acknowledgments}

This work was supported by Colciencias-Colombia, Grant No. 647 (2015 call for National PhD studies), and Région Bretagne in the framework of the Investissement d'Avenir Program through Labex CAMI (ANR-11-LABX-0004).

\section{Conflict of Interest}

The authors declare that they have no conflict of interest.

\section{References}

1. Achanta, R., Shaji, A., Smith, K., Lucchi, A., Fua, P., Susstrunk, S.: Slic superpixels compared to state-of-the-art superpixel methods. IEEE transactions on pattern analysis and machine intelligence 34(11), 2274-2282 (2012)

2. Badesch, D.B., Champion, H.C., Sanchez, M.A.G., Hoeper, M.M., Loyd, J.E., Manes, A., McGoon, M., Naeije, R., Olschewski, H., Oudiz, R.J.: Diagnosis and assessment of pulmonary arterial hypertension. Journal of the American College of Cardiology 54(1 Supplement), S55-S66 (2009)

3. Berndt, D.J., Clifford, J.: Using dynamic time warping to find patterns in time series. In: KDD workshop. vol. 10, pp. 359-370. Seattle, WA (1994)

4. Betancur, J., Simon, A., Langella, B., Leclercq, C., Hernández, A., Garreau, M.: Synchronization and registration of cine magnetic resonance and dynamic computed tomography images of the heart. IEEE journal of biomedical and health informatics 20(5), 1369-1376 (2016)

5. Burt, P., Adelson, E.: The laplacian pyramid as a compact image code. IEEE Transactions on communications 31(4), 532-540 (1983)

6. Dahlem, K., Michels, G., Kobe, C., Bunck, A.C., Ten Freyhaus, H., Pfister, R.: Diagnosis of cardiac transthyretin amyloidosis based on multimodality imaging. Clinical Research in Cardiology 106(6), 471-473 (2017)

7. Freeman, W.T., Adelson, E.H.: The design and use of steerable filters. IEEE Transactions on Pattern Analysis \& Machine Intelligence (9), 891-906 (1991) 
8. Grewenig, S., Zimmer, S., Weickert, J.: Rotationally invariant similarity measures for nonlocal image denoising. Journal of Visual Communication and Image Representation 22(2), 117-130 (2011)

9. Haugaa, K.H., Basso, C., Badano, L.P., Bucciarelli-Ducci, C., Cardim, N., Gaemperli, O., Galderisi, M., Habib, G., Knuuti, J., Lancellotti, P.: Comprehensive multi-modality imaging approach in arrhythmogenic cardiomyopathyan expert consensus document of the european association of cardiovascular imaging. European Heart Journal-Cardiovascular Imaging 18(3), 237-253 (2017)

10. Huang, X., Hill, N.A., Ren, J., Guiraudon, G., Boughner, D., Peters, T.M.: Dynamic 3d ultrasound and $\mathrm{mr}$ image registration of the beating heart. In: International Conference on Medical Image Computing and Computer-Assisted Intervention. pp. 171-178. Springer (2005)

11. Itti, L.: Quantifying the contribution of low-level saliency to human eye movements in dynamic scenes. Visual Cognition 12(6), 1093-1123 (2005)

12. James, A.P., Dasarathy, B.V.: Medical image fusion: A survey of the state of the art. Information Fusion 19, 4-19 (2014)

13. Kiss, G., Thorstensen, A., Amundsen, B., Claus, P., D'hooge, J., Torp, H.: Fusion of 3d echocardiographic and cardiac magnetic resonance volumes. In: 2012 IEEE International Ultrasonics Symposium. pp. 126-129. IEEE (2012)

14. Klein, S., Pluim, J.P., Staring, M., Viergever, M.A.: Adaptive stochastic gradient descent optimisation for image registration. International journal of computer vision 81(3), 227 (2009)

15. Ma, Y.L., Penney, G.P., Rinaldi, C.A., Cooklin, M., Razavi, R., Rhode, K.S.: Echocardiography to magnetic resonance image registration for use in image-guided cardiac catheterization procedures. Physics in medicine and biology 54(16), 5039 (2009)

16. Mizuguchi, Y., Oishi, Y., Miyoshi, H., Iuchi, A., Nagase, N., Oki, T.: The functional role of longitudinal, circumferential, and radial myocardial deformation for regulating the early impairment of left ventricular contraction and relaxation in patients with cardiovascular risk factors: a study with two-dimensional strain imaging. Journal of the American Society of Echocardiography 21(10), 1138-1144 (2008)

17. Nagueh, S.F., Chang, S.M., Nabi, F., Shah, D.J., Estep, J.D.: Imaging to diagnose and manage patients in heart failure with reduced ejection fraction. Circulation: Cardiovascular Imaging 10(4), e005615 (2017)

18. Olsen, F.J., Bertelsen, L., de Knegt, M.C., Christensen, T.E., Vejlstrup, N., Svendsen, J.H., Jensen, J.S., Biering-Sorensen, T.: Multimodality cardiac imaging for the assessment of left atrial function and the association with atrial arrhythmias. Circulation: Cardiovascular Imaging 9(10), e004947 (2016)

19. Perperidis, D., Mohiaddin, R.H., Rueckert, D.: Spatio-temporal free-form registration of cardiac mr image sequences. Medical image analysis 9(5), 441-456 (2005)

20. Puyol-Anton, E., Sinclair, M., Gerber, B., Amzulescu, M.S., Langet, H., De Craene, M., Aljabar, P., Piro, P., King, A.P.: A multimodal spatiotemporal cardiac motion atlas from $\mathrm{mr}$ and ultrasound data. Medical image analysis 40, 96-110 (2017)

21. Szasz, O.: On products of summability methods. Proceedings of the American Mathematical Society 3(2), 257-263 (1952)

22. Thorpe, S., Fize, D., Marlot, C.: Speed of processing in the human visual system. nature 381(6582), $520(1996)$

23. Tobon-Gomez, C., De Craene, M., Mcleod, K., Tautz, L., Shi, W., Hennemuth, A., Prakosa, A., Wang, H., Carr-White, G., Kapetanakis, S.: Benchmarking framework for myocardial tracking and deformation algorithms: An open access database. Medical image analysis $17(6), 632-648$ (2013)

24. Valsangiacomo Buechel, E.R., Mertens, L.L.: Imaging the right heart: the use of integrated multimodality imaging. European heart journal 33(8), 949-960 (2012)

25. Yushkevich, P.A., Piven, J., Hazlett, H.C., Smith, R.G., Ho, S., Gee, J.C., Gerig, G.: User-guided 3d active contour segmentation of anatomical structures: significantly improved efficiency and reliability. Neuroimage 31(3), 1116-1128 (2006)

26. Zhai, Y., Shah, M.: Visual attention detection in video sequences using spatiotemporal cues. In: Proceedings of the 14th ACM international conference on Multimedia. pp. 815824. ACM (2006) 
27. Zhang, W., Noble, J.A., Brady, J.M.: Spatio-temporal registration of real time 3d ultrasound to cardiovascular mr sequences. In: International Conference on Medical Image Computing and Computer-Assisted Intervention. pp. 343-350. Springer (2007)

28. Zhang, W., Noble, J.A., Brady, J.M.: Adaptive non-rigid registration of real time 3d ultrasound to cardiovascular mr images. In: Biennial International Conference on Information Processing in Medical Imaging. pp. 50-61. Springer (2007)

29. Zhao, N., Basarab, A., Kouamé, D., \& Tourneret, J. Y. Joint segmentation and deconvolution of ultrasound images using a hierarchical Bayesian model based on generalized Gaussian priors. IEEE transactions on Image Processing, 25(8), 3736-3750. (2016).

30. Atehortúa, A., Garreau, M., \& Romero, E. Fusion of 4D echocardiography and cine cardiac magnetic resonance volumes using a salient spatio-temporal analysis. In 13 th International Conference on Medical Information Processing and Analysis (Vol. 10572, p. 105721A). International Society for Optics and Photonics. (2017)

31. Klein, S., Staring, M., Murphy, K., Viergever, MA \& Pluim, JPW. elastix: A toolbox for intensity-based medical image registration. IEEE Trans Med Imag. pp. 196-205. (2010)

32. Kanai, T., Kadoya, N., Ito, K., Onozato, Y., Cho, S. Y., Kishi, K., \& Jingu, K. Evaluation of accuracy of B-spline transformation-based deformable image registration with different parameter settings for thoracic images. Journal of radiation research, 55(6), 11631170. (2014)

33. Ou, Y., Akbari, H., Bilello, M., Da, X., \& Davatzikos, C. Comparative evaluation of registration algorithms in different brain databases with varying difficulty: results and insights. IEEE transactions on medical imaging, 33(10), 2039-2065. (2014)

34. Klein, S., Staring, M., \& Pluim, J. P. Evaluation of optimization methods for nonrigid medical image registration using mutual information and B-splines. IEEE transactions on image processing, 16(12), 2879-2890. (2007)

35. Rueckert, D., Sonoda, L. I., Hayes, C., Hill, D. L., Leach, M. O., \& Hawkes, D. J. Nonrigid registration using free-form deformations: application to breast MR images. IEEE transactions on medical imaging, 18(8), 712-721. (1999) 